\title{
Riesgo de bacteriemia asociada a dispositivos intravasculares estratificados por peso de nacimiento en recién nacidos de un hospital público de alta complejidad: seguimiento de siete años
}

\author{
Patricio Nercelles, Sebastián Vernal, Pola Brenner y Pamela Rivero
}

\footnotetext{
Universidad de Valparaíso, Chile. Escuela de Medicina (PN, SV, PB) Hospital Carlos Van Buren, Valparaíso, Chile. Unidad de Prevención y Contro de Infecciones (PR).

Los autores declaran no tener conflictos de interés.

Sin financiamiento
}

Recibido: 9 de septiembre de 2014 Aceptado: 20 de diciembre de

Correspondencia a: Patricio Nercelles Muñoz patricio.nercelles@uv.c

\section{Risk of bacteremia associated with intravascular devices stratified by birth weight in born of a public hospital of high complexity: follow-up to seven years}

Introduction: Bacteremia associated to vascular catheters is the most frequent nosocomial infection in Neonatal Intensive Care Units and increases cost and mortality. Objective: To know the risk of bacteremia related to vascular devices in hospitalized newborns, stratified by birth weight. Method: A surveillance system was established considering birth weight and type of catheters in order to detect bacteremia and look for the risks associated to type of catheters and birth weight in the period 2005 to 2011, according to Chilean Ministry of Health's surveillance. Results: We registered bacteremia associated to vascular devices in newborns considering birth weight between less than $1,000 \mathrm{~g}$ to more than 2,500 g from years 2005 and 2011. In the period, 4,704 patients were surveyed with 25,130 catheter days and 70 bacteremia were detected. The rates of bacteremia were 0.9 per 1,000 catheter days in peripheral catheters, 3.0 per 1000 catheter days in peripheral inserted central catheters and 9.6 per 1,000 catheter days in umbilical catheters (UC). On the other side the risk of bacteremia was $6.4 \%$ in newborns with birth weight less $1,000 \mathrm{~g}$ and $1.5 \%$ in newborns with birth weight over 2,500 g. Coagulase negative Staphylococcus was the most frequent isolate agents. Conclusions: The risk of developing bacteremia is associated with lower birth weight and the use of UC independent of weight.

Key words: Nosocomial infection; catheter-related infection; bloodstream infection; surveillance; neonatal intensive care unit; newborns stratification weight.

Palabras clave: Infección nosocomial; Infección relacionada a catéter; bacteriemias; vigilancia; unidad cuidados intensivos neonatal; Estratificación peso nacimiento.

\section{Introducción}

L a bacteriemia asociada al uso de dispositivos intravasculares constituye la infección nosocomial más frecuente en las unidades de cuidado intensivo neonatal (UCIN), con prevalencia cercana a $30 \%$ en Estados Unidos de América ${ }^{1-3}$, una tasa de 1,1 por cada 1.000 días de uso de dispositivos durante el año $2010^{1}$, y una mortalidad asociada que va de 12 a $25 \%$ en pacientes críticos ${ }^{4}$.

En Chile durante el año 2011 las tasas de infecciones del torrente sanguíneo (bacteriemia/septicemia) en neonatos fueron de 3,5 por cada 1.000 días de exposición a catéter venoso central (CVC), 2,8 por cada 1.000 días de exposición a nutrición parenteral total (NPT) y 5,0 por cada 1.000 días de exposición a catéter umbilical $(\mathrm{CU})^{5}$. Estas infecciones aumentan los costos de la atención por una mayor estadía hospitalaria ${ }^{6-8}$.

Diferentes organizaciones han publicado datos res- pecto a la incidencia de bacteriemias en neonatos tales como el National Healthcare Safety Network (NHSN) y la International Nosocomial Infection Control Consortium (INICC) $)^{9,10}$.

La información nacional respecto a la incidencia de bacteremias en neonatos en nuestro país es aportada a través del sistema de vigilancia del Ministerio de Salud de Chile (MINSAL). El informe del año 2006 muestra que los agentes etiológicos notificados fueron Staphylococcus coagulasa negativa y Staphylococcus aureus en pacientes con CVC y CU, seguidos por Klebsiella pneumoniae en los pacientes con CVC, y por Enterobacter agglomerans en los pacientes con $\mathrm{CU}^{11}$. En estos informes no existen datos acerca de la bacteriemias estratificado de acuerdo al peso de nacimiento, a diferencia del CDC que lo estratifica en cinco grupos: ( $\leq 750$ gramos $(\mathrm{g}), 751-1.000 \mathrm{~g}, 1.001$ $1.500 \mathrm{~g}, 1.501-2.500 \mathrm{~g} \mathrm{y}>2.500 \mathrm{~g})^{9,11}$.

El objetivo de este trabajo es conocer el riesgo de bacteriemias relacionadas al uso de dispositivos intra- 
vasculares (catéter venoso periférico [CVP], catéter venoso central por vía percutánea $[\mathrm{CVCP}]$ y $\mathrm{CU}$ ) en recién nacidos internados en la UCIN de un hospital de alta complejidad, estratificados de acuerdo a su peso de nacimiento, utilizando las definiciones de vigilancia de infecciones del MINSAL.

\section{Material y Método}

Definición de bacteriemia asociada a dispositivos intravasculares: Se utilizaron las definiciones epidemiológicas entregadas por el MINSAL en el año 1998 para las bacteriemias asociadas al uso de los dispositivos intravasculares estudiados ${ }^{12}$.

Lugar de realización: Este estudio se realizó en la (UCIN) del Hospital Carlos Van Buren de Valparaíso.

Pacientes: Se recolectó la información de todos los pacientes ingresados a la UCIN desde enero del año 2005 hasta diciembre del año 2011. Se incorporaron al estudio aquellos pacientes con uso de CVP, CVCP y CU por al menos $24 \mathrm{~h}$ de duración, con vigilancia diaria de aparición de bacteriemia. El fin del seguimiento estaba dado por el retiro del catéter, alta, traslado de UCIN, o fallecimiento.

Los pacientes fueron analizados de acuerdo a su peso de nacimiento, separándolos en cuatro grupos en correlación con los reportes de la NHSN previos al año 2006. Los grupos son los siguientes: 1) $\leq 1.000 \mathrm{~g}$; 2) 1.001-1.500 g; 3) $1.501-2.500 \mathrm{~g} \mathrm{y} 4)>2.500 \mathrm{~g}$.

Tipo de estudio: Cohorte prospectiva en la que se analizaron las siguientes variables:

- Tipo de catéter utilizado.

a. Catéter venoso periférico.

b. Catéter venoso central por vía percutánea.

c. Catéter umbilical.

- Días de uso de cada catéter.

- Bacteriemias asociadas al uso de cada catéter.

- Microbiología de las bacteriemias.

- Peso de nacimiento del neonato.

Comité Ético: La privacidad de cada paciente fue protegida y codificada para la recolección de información, y forma parte de la vigilancia epidemiológica habitual del hospital.

Análisis de datos: Para la organización de datos y análisis estadístico se utilizó el programa Microsoft Excel 200712.0 (Microsoft Office 2007, Microsoft Corporation). El estudio estadístico se realizó mediante cálculo de riesgo relativo e intervalo de confianza de $95 \%$ para la comparación de riesgo de bacteriemias asociadas al tipo de dispositivo intravascular y el peso de nacimiento.

\section{Resultados}

Análisis descriptivo de las variables

Desde enero de 2005 a diciembre de 2011 fueron incorporados 4.704 pacientes con utilización de CVP, CVCP y CU, con un total de 25.130 días de uso de catéter. Los CU fueron utilizados por 3.421 días, con una duración promedio de 4,9 días; los CVP se utilizaron en 3.098 pacientes por 13.505 días con una duración promedio de 4,4 días y los CVCP se utilizaron en 915 recién nacidos por 8.204 días, con una duración promedio de 8,9 días. No se pudo calcular la mediana y rango de utilización de los catéteres por tratarse de datos consolidados de la vigilancia.

La distribución de los pacientes categorizados de acuerdo al peso de nacimiento fue la siguiente: 682 pacientes pesaron $1.000 \mathrm{~g}$ o menos, 871 pesaron entre $1.001 \mathrm{~g}$ y $1.500 \mathrm{~g}, 1.482$ pesaron entre $1.501 \mathrm{~g}$ y $2.500 \mathrm{~g}$ y 1.669 pesaron más de $2.500 \mathrm{~g}$.

Se identificaron 70 bacteriemias asociadas al uso de los dispositivos intravasculares estudiados, de las cuales 33 bacteriemias fueron asociadas al uso de CU (47\%), 12 bacteriemias se asociaron al uso de CVP (12\%) y 25 bacteriemias se asociaron al uso de CVCP (36\%). La mitad de las bacteriemias se produjeron en los niños con peso de nacimiento menor a $1.000 \mathrm{~g}$ (Tabla 1).

El mayor riesgo de bacteriemia se observó en los neonatos con CU (9,6 por 1.000 días catéter) y el menor en los que utilizaron CVP (0,9 por 1.000 días catéter) (Tabla 2). El riesgo de bacteriemia fue significativamente mayor en los pacientes que utilizaron $\mathrm{CU}$ versus $\mathrm{CVCP}$ y CVP (RR: 5.66) (Tabla 3).

Tabla 1. Proporción de bacteriemias según tipo de catéter y peso de nacimiento

\begin{tabular}{|c|c|c|c|c|c|c|}
\hline $\begin{array}{l}\text { Tipo de } \\
\text { catéter }\end{array}$ & $\leq 1.000 \mathrm{~g}$ & $\begin{array}{l}1.001 \mathrm{a} \\
1.500 \mathrm{~g}\end{array}$ & $\begin{array}{l}1.501 \mathrm{a} \\
2.500 \mathrm{~g}\end{array}$ & $>2.500 \mathrm{~g}$ & Total & $\%$ \\
\hline CVP & 0 & 5 & 4 & 3 & 12 & 17 \\
\hline CVCP & 13 & 3 & 7 & 2 & 25 & 36 \\
\hline CU & 22 & 5 & 1 & 5 & 33 & 47 \\
\hline Total & 35 & 13 & 12 & 10 & 70 & 100 \\
\hline Porcentaje & 50 & 19 & 17 & 14 & 100 & \\
\hline
\end{tabular}

CVP: catéter venoso periférico. CVCP: catéter venoso central por vía percutánea. CU: catéter umbilical.

\begin{tabular}{|cccc|}
\hline \multicolumn{5}{c}{ Tabla 2. Riesgo de bacteriemia por tipo de catéter } \\
\hline Catéter & Bacteremias $(\mathbf{n})$ & Días catéter & Tasa \%o \\
\hline CVP & 12 & 13.505 & 0,9 \\
\hline CVCP & 25 & 8.204 & 3,0 \\
\hline CU & 33 & 3.421 & 9,6 \\
\hline Total & 70 & 25.130 & 2,8 \\
\hline
\end{tabular}




\begin{tabular}{|l|cc|}
\hline Tabla 3. Comparación de riesgo de bacteriemia por tipo \\
de catéter
\end{tabular}

Tabla 4. Riesgo de bacteriemia por tipo de catéter y peso de nacimiento

\begin{tabular}{lccccc} 
& $\begin{array}{c}\text { Pacientes } \\
\mathbf{S 1 . 0 0 0} \mathbf{g}\end{array}$ & $\begin{array}{c}\text { Bacteremias } \\
\mathbf{( n )}\end{array}$ & $\begin{array}{c}\text { Días } \\
\text { catéter }\end{array}$ & $\begin{array}{c}\text { Promedio días } \\
\text { por paciente }\end{array}$ & $\begin{array}{c}\text { Tasa } \\
\% \mathbf{o}\end{array}$ \\
CVP & 268 & 0 & 1.531 & 5,7 & 0,0 \\
CVCP & 219 & 13 & 2.727 & 12,5 & 4,8 \\
CU & 195 & 22 & 1.202 & 6,2 & 18,3 \\
Total & 682 & 35 & 5.460 & 8,0 & 6,4 \\
$\mathbf{1 . 0 0 1 - 1 . 5 0 0 ~ g}$ & & & & & \\
CVP & 346 & 3 & 1.677 & 4,8 & 1,8 \\
CVCP & 338 & 5 & 3.026 & 9,0 & 1,7 \\
CU & 187 & 5 & 939 & 5,0 & 5,3 \\
Total & 871 & 13 & 5.642 & 6,5 & 2,3 \\
$\mathbf{1 . 5 0 1 - 2 . 5 0 0 ~ g}$ & & & & & \\
CVP & 1.112 & 7 & 5.235 & 4,7 & 1,3 \\
CVCP & 218 & 4 & 1.606 & 7,4 & 2,5 \\
CU & 152 & 1 & 534 & 3,5 & 1,9 \\
Total & 1.482 & 12 & 7.375 & 5,0 & 1,6 \\
> 2.500 g & & & & & \\
CVP & 1.372 & 2 & 5.062 & 3,7 & 0,4 \\
CVCP & 140 & 3 & 845 & 6,0 & 3,6 \\
CU & 157 & 5 & 746 & 4,8 & 6,7 \\
Total & 1.669 & 10 & 6.653 & 4,0 & 1,5 \\
\hline
\end{tabular}

\begin{tabular}{|}
\begin{tabular}{|lcc|}
\hline Tabla 5. Comparación de riesgo de bacteriemia por peso \\
de nacimiento
\end{tabular} \\
\begin{tabular}{|lcc} 
Peso de nacimiento & $\begin{array}{c}\text { Riesgo } \\
\text { relativo }\end{array}$ & $\begin{array}{c}\text { Intervalo de } \\
\text { confianza } 95 \%\end{array}$ \\
\hline$\leq 1.000 \mathrm{vs}>2.500 \mathrm{~g}$ & 4,26 & $3,56-4,97$ \\
$1.001-1.500 \mathrm{vs}>2.500 \mathrm{~g}$ & 1,53 & $0,71-2,36$ \\
\hline $1.501-2.500$ vs $>2.500 \mathrm{~g}$ & 1,08 & $0,24-1,92$ \\
\hline
\end{tabular}
\end{tabular}

\begin{tabular}{|c|c|c|}
\hline Grupos & $\begin{array}{l}\text { Riesgo } \\
\text { relativo }\end{array}$ & $\begin{array}{c}\text { Intervalo de } \\
\text { confianza } 95 \%\end{array}$ \\
\hline$\leq 1.000 \mathrm{~g} \mathrm{CU}$ vs CVCP & 3,84 & $3,15-4,53$ \\
\hline CVP y CVCP $\leq 1.000$ vs $>2.500 \mathrm{~g}$ & 3,59 & $3,05-4,62$ \\
\hline$C U \leq 1.000$ vs $>2.500 \mathrm{~g}$ & 2,73 & $2,25-3,70$ \\
\hline
\end{tabular}

En la Tabla 4 se observa el comportamiento de las tasas de bacteriemias de acuerdo a la categorización de los pacientes por peso de nacimiento y considerando el uso de todos los tipos de dispositivos vasculares. Los neonatos con peso $\leq 1.000 \mathrm{~g}$ tuvieron una tasa de 6,4 por cada 1.000 días catéter, siendo esta tasa significativamente mayor comparado con los neonatos de peso mayor a $2.500 \mathrm{~g}(\mathrm{RR} 4,26)$. En los neonatos con peso entre 1.001-1.500 g se presentaron 13 bacteriemias con tasa de 2,3 por cada 1.000 días catéter, no se observaron diferencias significativas al compararlas con los neonatos de peso mayor a $2.500 \mathrm{~g}(\mathrm{RR} 1,53)$. En los neonatos con peso entre 1.501-2.500 g hubo 12 bacteriemias con tasa de 1,63 y en los neonatos de $>2.500 \mathrm{~g}$ la tasa fue de 1,5 con 10 bacteriemias detectadas. Se observó mayor riesgo de bacteriemias en neonatos con peso $\leq$ a $1.000 \mathrm{~g}$ tanto en catéteres insertados por vía periférica (RR 3,59), como en catéteres umbilicales (RR 2,73) en comparación a los neonatos con peso mayor a $2.500 \mathrm{~g}$ (Tablas 5 y 6).

En la Tabla 7 se observa la distribución de los microorganismos de acuerdo al tipo de dispositivo utilizado. De los patógenos aislados, el agente más frecuente fue Staphylococcus coagulasa negativa $(46 \%)$, seguido por $K$. pneumoniae $(20 \%)$ y $S$. aureus $(10 \%)$. El principal microorganismo aislado con el uso de CVP fue $S$. aureus (5 casos) seguido de K. pneumoniae y Staphylococcus coagulasa negativa ( 3 casos cada uno de ellos). En tanto en las bacteriemias asociadas a CU y CVCP el microorganismo principalmente aislado fue Staphylococcus coagulasa negativa causando 16 y 13 bacteriemias respectivamente, seguido de $K$. pneumoniae con 3 y 8 casos respectivamente.

\section{Discusión}

Este estudio es el primero realizado en Chile que compara tasas de bacteriemia en neonatos estratificadas por peso de nacimiento de manera similar a los datos reportados por la NHSN.

Respecto al desarrollo de bacteriemias asociado al uso de CU, si comparamos nuestros resultados con los datos reportados a través de los años por la NHSN, nuestra tasa se encuentra sobre el percentil 90 en comparación con los reportes entre los años 2006 y $2010^{1,9,13-15}$. No es posible comparar estos resultados con los del MINSAL ya que las tasas publicadas no están estratificadas por peso de nacimiento.

En este estudio se observa claramente que existe un riesgo mayor de desarrollar bacteriemia en $\mathrm{RN}$ con peso de nacimiento de $1.000 \mathrm{~g}$ o menos tanto para el uso de CVCP como CU en comparación a CVP. Sin embargo, en los niños con peso $1.000 \mathrm{~g}$ o menos, no se registraron bacteriemias tras el uso de CVP durante 


\begin{tabular}{|c|c|c|c|c|c|c|c|c|}
\hline \multirow[t]{2}{*}{ Microorganismo } & \multirow{2}{*}{\multicolumn{2}{|c|}{ Catéter venoso periférico }} & \multicolumn{2}{|c|}{ Catéter venoso central vía percutánea } & \multicolumn{2}{|c|}{ Catéter umbilical } & \multicolumn{2}{|c|}{ Total } \\
\hline & & & $\mathbf{n}$ & $\%$ & $\mathbf{n}$ & $\%$ & $n$ & $\%$ \\
\hline Staphylococcus coagulasa negativa & 3 & 25 & 17 & 68 & 15 & 45,5 & 35 & 50 \\
\hline Klebsiella pneumoniae & 3 & 25 & 3 & 12 & 8 & 24,2 & 14 & 20 \\
\hline Staphylococcus aureus & 5 & 42 & 1 & 4 & 1 & 3 & 7 & 10 \\
\hline Enterobacter aerogenes & 1 & 8 & 1 & 4 & 3 & 9,1 & 5 & 7,1 \\
\hline Pseudomonas aeruginosa & - & - & 2 & 8 & 2 & 6,1 & 4 & 5,7 \\
\hline Enterobacter cloacae & - & - & - & - & 2 & 6,1 & 2 & 2,9 \\
\hline Escherichia coli & - & - & 1 & 4 & - & - & 1 & 1,4 \\
\hline Citrobacter freundii & - & - & - & - & 1 & 3 & 1 & 1,4 \\
\hline Enterococcus faecium & - & - & - & - & 1 & 3 & 1 & 1,4 \\
\hline Total & 12 & & 25 & & 33 & & 70 & 100 \\
\hline
\end{tabular}

nuestro seguimiento, además en este grupo se observó la mayor utilización de CU (35\% de los días catéter). Estos resultados, son concordantes con lo publicado por la NHSN. Llama la atención que en nuestro estudio en ese estrato, no se presentaron bacteriemias en pacientes con catéter periférico, por lo que en futuros estudios debería dilucidarse si el factor de riesgo corresponde a bajo peso, al uso de CU o a ambos.

Se evidenció además que existe un riesgo más elevado de bacteriemias en los neonatos al utilizar CU y CVCP comparado con CVP. Es evidente que la utilización de CU como vía de acceso intravascular se asocia a un elevado riesgo de desarrollar bacteriemias en comparación con los otros dispositivos, por lo que su indicación debería estar reservada sólo a neonatos que por su condición no sea viable otra vía de acceso. Esta asociación también ha sido descrita en estudios prospectivos, donde se estima un mayor riesgo de bacteriemias tras el uso de CU (Hazard Ratio 1,79) versus otros dispositivos por vía venosa central ${ }^{16}$.

Los microorganismos aislados fueron principalmente cocáceas grampositivas, siendo el más frecuente Staphylococcus coagulasa negativa en las bacteriemias asociadas al uso de CVCP y CU, seguido de $S$. aureus que tuvo una mayor presencia en desarrollo de bacteriemias asociadas a CVP; tendencia similar se describe en los reportes de la NHSN y en otros estudios ${ }^{1,17,18}$. Dentro de los bacilos gramnegativos, más frecuente fue $K$. pneumoniae en cualquiera de los tipos de dispositivos intravasculares estudiados. En ninguno de los neonatos que ingresaron a la vigilancia de este estudio se evidenciaron infecciones por Candida sp., a diferencia de otros reportes ${ }^{1,2,3,9}$, probablemente por menor utilización de antimicrobianos en la Unidad, asociado a políticas de racionalización de antimicrobianos del hospital.

\section{Conclusiones}

En la Unidad Neonatal del hospital se utilizan principalmente tres vías para acceso intravasculares en $\mathrm{RN}$, siendo el CP la vía más utilizada; pese a ello se observó una mayor incidencia de bacteriemias tras el uso de $\mathrm{CU}$ versus CVP y CVCP.

El riesgo de desarrollar bacteriemia se asocia a menor peso de nacimiento y a la utilización de $\mathrm{CU}$, independientemente del peso. Staphylococcus coagulasa negativa fue el agente mas frecuentemente aislado en bacteriemia en todos los grupos estudiados.

Este trabajo demuestra que en neonatos los riesgos de bacteriemia difieren significativamente dependiendo del tipo de catéter utilizados y del peso de nacimiento, lo que debería ser considerado cuando se realizan comparaciones entre hospitales de distinta complejidad y para el establecimiento o ajustes de sistemas de vigilancia epidemiológica de bacteriemias en este grupo de pacientes.

Agradecimientos. Al personal de la Unidad de Neonatología y del Laboratorio de Microbiología, que con su trabajo diario permitieron contar con mucha de la información de este trabajo.

\section{Resumen}

Introducción: La bacteriemia asociada al uso de dispositivos intravasculares constituye la infección nosocomial más frecuente en las unidades de cuidado intensivo neonatal, asociándose a mayores costos y letalidad. Objetivo: Conocer el riesgo de bacteriemias relacionadas al uso de dispositivos intravasculares en recién nacidos hospitalizados, de acuerdo a su peso de nacimiento. Material y 
Métodos: Vigilancia epidemiológica de bacteriemia en neonatos con catéteres vasculares para asociarlos con los tipos de catéteres y el peso de nacimiento, durante el período 2005 a 2011, utilizando el sistema de vigilancia vigente en el país. Resultados: Se registraron las bacteriemias asociadas a dispositivos vasculares en neonatos considerando peso de nacimiento entre menos a $1.000 \mathrm{~g}$ a más de $2.500 \mathrm{~g}$ entre los años 2005 y 2011. Se vigilaron 4.704 pacientes con 25.130 días de uso de catéter y se detectaron 70 bacteriemias. La mayor utilización de la vía venosa fue por catéteres venosos periféricos. Las tasas de bacteriemias fueron de 0,9 por mil días catéter en venoso periférico, 3,0 en catéter central por vía percutánea y 9,6 en catéter umbilical (CU). El riesgo de bacteriemia fue de $6,4 \%$ en los neonatos de peso $<1.000 \mathrm{~g} \mathrm{y} 1,5 \%$ o en $>2.500$ g. Staphylococcus coagulasa negativa fue el agente más frecuentemente aislado. Conclusiones: El riesgo de desarrollar bacteriemia se asoció a menor peso de nacimiento y a la utilización de CU en forma, independiente del peso.

\section{Referencias bibliográficas}

1.- Dudeck M, Horan T, Peterson K, Allen-Bridson K, Morell G, Pollock D, et al. National Healthcare Safety Network (NHSN). National Healthcare Safety Network Report Data Summary 2010. www.cdc.gov.

2.- Stoll B J, Hansen N, Fanaroff A A, Wright L L, Carlo W A, Ehrenkranz R A, et al. Late-onset BSI in very low birth weight neonates: the experience of the NICHD Neonatal Reserch Network. Pediatrics 2002; 110: 285-91.

3.- Stover B H, Shulman S T, Bratcher D F, Brady M T, Levine G L, Jarvis W R. Nosocomial infection rates in US children's hospital neonatal and pediatric intensive care units. Am J Infect Control 2001; 29: 152-7.

4.- $\mathrm{O}^{\prime}$ Grady N P, Alexander M, Dellinger E P, Gerberding J, Heard S, Maki D, et al. Guidelines for the prevention of intravascular catheter-related infections. MMWR Recomm Rep 2002; 51: 1-29.

5.- Subsecretaría de redes asistenciales, Departamento de Calidad y Seguridad del Pacientes. Indicadores de Infecciones asociadas a la atención de salud año 2011. http://web. minsal.cl/portal/url/item/8794c42f35ad5111e04 001011e013f93.pdf (Acceso 20/08/2014).

6.- Brenner P, Nercelles P, Pohlenz M, Otaíza F. Costo de las infecciones intrahospitalarias en hospitales chilenos de alta complejidad y mediana complejidad. Rev Chilena Infectol 2003; 20 (4): 285-90.

7.- Warren D K, Quadir W W, Hollenbeak C S, Elward A M, Cox M J, Fraser V J. Attributable cost of catheter-associated bloodstream infections among intensive care patients in a nonteaching hospital. Crit Care Med 2006; 34 : 2084-9.

8.- Rosenthal V D, Guzmán S, Migone O, Safdar N. The attributable cost, length of hospital stay, and mortality in central-line associated bloodstream infection in intensive care departments in Argentina: a prospective, matched analysis. Am J Infect Control 2003; 31: 475-80.

9.- Edwards J R, Peterson K D, Andrus M L, Dudeck M, Pollock D, Horan T, et al. National Healthcare Safety Network (NHSN). National Healthcare Safety Network Report Data Summary for 2006. www.cdc.gov.

10.- Rosenthal V D, Bijie H, Maki D G, Mehta Y, Apisarnthanarak A, Medeiros E A, et al. International Nosocomial Infection Control Consortium (INICC) report, data summary of 36 countries, for 2004-2009. Amer J Infect Control 2011; 30: 1-11.

11.- Bustamante R, Espínola V. Informe de Vigilancia Epidemiológica de Infecciones Intrahospitalarias Chile, 2007. http://web. minsal.cl/portal/url/item/73627aa2edca0374e04 001011f01734d.pdf. (Acceso el 20 de agosto de 2014).

12.- Ministerio de Salud. Sistema de vigilancia de las infecciones intrahospitalarias, año 1998. http:/web.minsal.cl/portal/url/item/8a331613f0 71f5e7e04001011e010f96.pdf (Acceso el 20 de agosto de 2014).

13.- Edwards J R, Peterson K D, Andrus M L, Tolson J S, Goulding J S, Dudeck M A, et al. National Healthcare Safety Network (NHSN). National Healthcare Safety Network Report Data Summary for 2007. www.cdc.gov. 14.- Edwards J R, Peterson K D, Mu Y, Banerjee S,
Allen-Bridson K, Morrell G, et al. National Healthcare Safety Network (NHSN). National Healthcare Safety Network Report Data Summary for 2008. www.cdc.gov.

15.- Dudeck M A, Horan T C, Peterson K D, Bridson K A, Morell G C, Pollock D A, et al. National Healthcare Safety Network (NHSN). National Healthcare Safety Network Report Data Summary for 2009. www.cdc.gov.

16.- Van der Zwet W C, Kaiser A M, Van Elburg R M, Berkhof J, Fetter W P, Parlevliet G A, et al. Nosocomial infections in a Dutch neonatal intensive care unit: surveillance study with definitions for infection specifically adapted for neonates. J Hosp Infect 2005; 61: 300-11.

17.- Rosenthal V D, Lynch P, Jarvis W R, Khader I A, Richtmann R, Jaballah N B, et al. Socioeconomic impact on device-associated infections in limited-resource neonatal intensive care units: finding of the INICC. Infection 2011; 39: 439-50.

18.- Urrea M, Pons M, Serra M, Latorre C, Palomeque A. Prospective incidence study of nosocomial infections in a pediatric intensive care unit. Pediatr Infect Dis J 2003; 22: 490-3.

19.- Almuneef M A, Memish Z A, Balkhy H H, Hijazi O, Cunningham G, Francis C. Rate, risk factors and outcomes of catheter-related bloodstream infection in a paediatric intensive care unit in Saudi Arabia. J Hosp Infect 2006; 62: 207-13.

20.- Dueñas L, Bran A, Rosenthal V D, Machuca L. Device-associated infection rates in pediatric and neonatal intensive care units El Salvador: Findings of INICC. J Infect Dev Ctries 2011; 5 (6): 445-51. 